\title{
EQUIVALENCE CLASSES OF IDEALS IN THE NILRADICAL OF A BOREL SUBALGEBRA
}

\author{
ERIC N. SOMMERS
}

\section{Dedicated to George Lusztig on his 60th birthday}

\begin{abstract}
An equivalence relation is defined and studied on the set of $B$-stable ideals in the nilradical of the Lie algebra of a Borel subgroup $B$. Techniques are developed to compute the equivalence relation and these are carried out in the exceptional groups. There is a natural partial order on equivalence classes coming from inclusion of one ideal in another. A main theorem is that this partial order is a refinement of the closure ordering on nilpotent orbits.
\end{abstract}

\section{$\S 1$. Introduction}

Let $G$ be a connected simple algebraic group over $\mathbf{C}$ and $B$ a Borel subgroup of $G$. Let $\mathfrak{g}$ be the Lie algebra of $G$ and $\mathfrak{b}$ the Lie algebra of $B$. The nilradical of $\mathfrak{b}$ is denoted $\mathfrak{n}$.

The subspaces of $\mathfrak{n}$ which are stable under the adjoint action of $B$ (or the adjoint action of $\mathfrak{b}$ ) are ideals in $\mathfrak{n}$. They are called $B$-stable ideals of $\mathfrak{n}$ or often just called ideals. The study of these ideals has attracted much recent attention, both from the perspective of representation theory and also from the combinatorial perspective. In addition there are tantalizing connections to the numerology which arises in the theory of cluster algebras introduced by Fomin and Zelevinsky. We mention the work of Kostant, Cellini-Papi, Cellini-Frajria-Papi, Panyushev, Panyushev-Röhrle, Suter, Athanasiadis, Athanasiadis-Reiner, and the author.

Some of these works have dealt with connections to nilpotent orbits (for example, see [14]). It is worthwhile to note that a number of important earlier papers have exploited the set of ideals in order to study nilpotent orbits and the representation theory that depends upon them. We mention the

Received July 21, 2005.

Revised January 17, 2006.

2000 Mathematics Subject Classification: Primary 17B20; Secondary 20 F55.

Supported by NSF grants DMS-0201826 and DMS-0502254. 
following sources: Mizuno [13], DeConcini-Lusztig-Procesi [5], Kawanaka [8], and Shi [15]. The ideas in this paper were influenced by and expand upon the ideas in the first three of these papers.

Consider the following question. Given a $B$-stable ideal $I$, what is the $G$-saturation $G . I$ of $I$ where

$$
G . I:=\{g . X \mid g \in G \text { and } X \in I\}
$$

where the dot refers to the adjoint action of $G$ on $\mathfrak{g}$, and how can this be computed? The saturation is necessarily the closure of a single nilpotent orbit, denoted by $\mathcal{O}_{I}$. From the Jacobson-Morozov theorem, it is clear that every orbit is equal to $\mathcal{O}_{I}$ for some ideal $I$.

On the other hand, it may be relevant to study the centralizer $B_{e}$ for $e \in \mathcal{O}_{I} \cap I$. However, $\mathcal{O}_{I} \cap I$ need not be a single $B$-orbit. The structure of the different centralizers can be captured by considering the moment map

$$
\pi: G \times{ }^{B} I \longrightarrow G . I
$$

given by $(g, X) \rightarrow g . X$. In some cases this map has connected fiber over a point in $\mathcal{O}_{I}$ (for example when $I$ arises from the Jacobson-Morozov theorem), but in other cases the fiber may be disconnected. Let $e \in I \cap \mathcal{O}_{I}$ and let $G_{e}$ denote the centralizer of $e$ in $G$. One can show as in [5] that the fiber $\pi^{-1}(e)$ is a disjoint union of irreducible smooth varieties and that $G_{e}$ acts transitively on the set of irreducible components. The identity subgroup $G_{e}^{0}$ clearly stabilizes each component. Let $H_{I}$ denote the stabilizer in $G_{e}$ of an irreducible component of the fiber. Then the image of $H_{I}$ in the component group

$$
A(e):=G_{e} / G_{e}^{0}
$$

defines a subgroup $K_{I}$ that is well-defined up to conjugacy, as in [5]. One of our goals is to understand for each ideal how to compute both $\mathcal{O}_{I}$ and $K_{I}$.

Note that if a subgroup $P$ containing $B$ stabilizes $I$, then one can define the analogous moment map. The saturation of $I$ will clearly be the same, but so too will the analogous subgroup $K_{I}$. In this more general setting, the subgroups $K_{I}$ have been studied (see [10]) when $I$ is the nilradical $\mathfrak{n}_{P}$ of the Lie algebra of any parabolic subgroup $P$ or more generally when $I$ arises from induction of an ideal coming from the Jacobson-Morozov ideal in a Levi subalgebra. Computing the saturation of such ideals amounts 
to calculating Lusztig-Spaltenstein induction [12]. See [20] for Elashvili's calculations in the exceptional groups and [4] for the classical groups.

The smallest interesting example occurs in type $B_{2}$. Here there are six ideals. Of these, one has saturation the zero orbit; one, the regular orbit; and one, the minimal orbit. The remaining three have saturation equal to the subregular orbit. For one of these ideals, the fiber over $e \in \mathcal{O}_{I}$ is connected, but for the other two, the fiber has two components. Hence in the former case, $K_{I}=S_{2}$ and in the latter cases, $K_{I}$ is trivial.

In order to study these questions, we define an equivalence relation on the set of $B$-stable ideals in $\mathfrak{n}$. It has the property that equivalent ideals have the same $\mathcal{O}_{I}$ and the same group $K_{I}$, which we henceforth regard as being defined up to conjugacy (and this is well-defined, independent of which base point $e$ we choose). The equivalence relation is closely related to the equivalence relation considered in [5] on a certain subset of ideals, or rather their intersection with the space $\mathfrak{g}_{2}$, arising from the JacobsonMorozov theorem for $e$. It is also strongly related to the work in [13], where Mizuno was interested in those ideals which have the same saturation and in simple operations on ideals which preserve the saturation.

We show that the natural partial order on ideals given by inclusion of one ideal in another ideal descends to give a partial order on equivalence classes. A main result is that this partial order refines the closure order on nilpotent orbits, something which is essentially proved in a case-by-case fashion by Mizuno [13] in the exceptional groups.

One issue which arises for each orbit $\mathcal{O}$ is the minimal value of the dimension of an ideal $I$ with $\mathcal{O}_{I}=\mathcal{O}$. Kawanaka [8] computed a minimal possible value for the dimension of $I$ and then used Mizuno's work to show that the minimal value is always obtained for each orbit in the exceptional groups. We extend Kawanaka's work by giving a conjectural algorithm to find an ideal achieving this minimal value. These ideas are then employed to compute $\mathcal{O}_{I}$ and $K_{I}$ for each ideal $I$ in the exceptional groups and thus the partial order on the set of equivalence classes of ideals.

The paper concludes with some speculations. In particular we speculate on a connection between the partial order on equivalence classes and the partial order introduced by Achar [1] on the set of pairs consisting of a nilpotent orbit and a conjugacy class in Lusztig's canonical quotient. We show that these conjectures are true in the exceptional groups.

It is a pleasure to dedicate this paper to Professor Lusztig, my Ph.D. advisor. I have been greatly influenced by his ideas and perspectives, gleaned 
both from his many beautiful papers and lectures, as well as from numerous conversations over the past decade.

\section{$\S 2$. Definitions}

Let $G / B$ be the flag manifold of $G$. For a rational representation of $\tau: B \rightarrow G L(V)$, we can form the vector bundle $G \times{ }^{B} V$ over $G / B[21]$. For convenience, we use $V$ also to denote the sheaf of sections of this bundle and write $H^{i}(G / B, V)$ for its cohomology groups. Fix a maximal torus $T$ in $B$ and let $\left(X, \Phi, Y, \Phi^{\vee}\right)$ be the root datum determined by $G$ and $T$, and let $W$ be the Weyl group. Let $\Pi \subset \Phi^{+}$denote the simple roots and positive roots determined by $B$. As usual, $\langle$,$\rangle denotes the pairing of X$ and $Y$.

We denote the standard partial order on $\Phi$ by $\preceq$; so $\alpha \preceq \beta$ for $\alpha, \beta \in \Phi$ if and only if $\beta-\alpha$ is a sum of positive roots.

We define an ideal $\mathcal{I}$ of $\Phi^{+}$to be a collection of roots such that if $\alpha \in \mathcal{I}$, $\beta \in \Phi^{+}$, and $\alpha+\beta \in \Phi^{+}$, then $\alpha+\beta \in \mathcal{I}$. In other words, if $\alpha \in \mathcal{I}$ and $\gamma \in \Phi^{+}$with $\alpha \preceq \gamma$, then $\gamma \in \mathcal{I}$.

It is easy to see that $B$-stable ideals in the nilradical $\mathfrak{n}$ of $\mathfrak{b}$ are naturally in bijection with the ideals of $\Phi^{+}$. Namely, if $I$ is a $B$-stable ideal of $\mathfrak{n}$, it is stable under the action of $T$, hence $I$ is a sum of roots spaces. Denote by $\mathcal{I}$ the set of roots whose root space is contained in $I$. Then $\mathcal{I}$ is an ideal of $\Phi^{+}$, called the support of $I$, and this map is a bijection. We will often use $I$ both for an ideal in $\mathfrak{n}$ and for the corresponding ideal in $\Phi^{+}$.

Given an ideal $\mathcal{I}$ in $\Phi^{+}$, we define the minimal roots $\mathcal{I}_{\text {min }}$ of $\mathcal{I}$ as follows: $\alpha \in \mathcal{I}$ belongs to $\mathcal{I}_{\text {min }}$ if and only if for all $\beta \in \Phi^{+}$with $\beta \preceq \alpha$ and $\beta \neq \alpha$, then $\beta \notin \mathcal{I}$. Clearly $\mathcal{I}$ determines and is determined by its set of minimal roots $\mathcal{I}_{\text {min }}$. Note that the elements of $\mathcal{I}_{\text {min }}$ are mutually incomparable elements of $\Phi^{+}$and that every set of mutually incomparable elements of $\Phi^{+}$is an $\mathcal{I}_{\text {min }}$ for a unique $\mathcal{I}$ (namely, $\mathcal{I}$ is the set of all elements bigger or equal to the elements of $\left.\mathcal{I}_{\text {min }}\right)$.

We are ready to define an equivalence relation on the ideals of $\mathfrak{n}$. If $V_{1} \subset V_{2}$ are representations of $B$, then the restriction of the polynomials of degree $n$ on $V_{2}$ to polynomials on $V_{1}$ induces a map in cohomology:

$$
H^{i}\left(G / B, S^{n} V_{2}^{*}\right) \longrightarrow H^{i}\left(G / B, S^{n} V_{1}^{*}\right)
$$

where $S^{n}\left(-^{*}\right)$ denotes the $n$-th symmetric power of the linear dual. More precisely, this map arises from the Koszul resolution of the ideal defining $V_{1}$ in $V_{2}$. For ideals of $\mathfrak{n}$ we make the definition 
Definition 2.1. Let $I \subseteq J$ be two ideals of $\mathfrak{n}$. Then $I$ and $J$ are equivalent if the map in (1) is an isomorphism for all $n \geq 0$ whenever $i=0$.

The reason we are ignoring the higher cohomology groups (in contrast with [2]) is that it seems likely that these cohomology groups vanish for ideals whenever $i>0$ and also it is harder to establish some of the basic properties of the equivalence relation if we require at this early stage that (1) is an isomorphism for all $i \geq 0$.

Note that if $I_{1} \subseteq I_{2} \subseteq I_{3}$ are ideals, then $I_{1}$ and $I_{3}$ are equivalent if and only if both $I_{1}$ and $I_{2}$ are equivalent and $I_{2}$ and $I_{3}$ are equivalent. Thus it is clear that the above definition generates a well-defined equivalence relation on the set of ideals in $\mathfrak{n}$. We write $I \sim J$ for this relation.

Now it is not hard to see that if $I \subseteq J$ are ideals, then there exist a chain of ideals

$$
I=I_{0} \subseteq I_{1} \subseteq \cdots \subseteq I_{k}=J
$$

such that the $I_{m} / I_{m-1}$ is one-dimensional. Indeed, $I_{m-1}$ is obtained from $I_{m}$ by omitting a root space corresponding to a minimal root in the support of $I_{m}$ (which does not belong to the support of $I$ ). In this way the $I_{m}$ are inductively defined and the process clearly terminates in $I$ after a finite number of steps. Consequently if $I$ and $J$ are equivalent, all the ideals $I_{m}$ lie in the same equivalence class. Thus in order to describe an equivalence class of ideals, we are often led to focus on ideals $I \subseteq J$ such that $J / I$ is one-dimensional.

Let us assume that this is the case and write $U=J / I$ for the onedimensional $B$-representation. The Koszul resolution for the exact sequence of $B$-modules

$$
0 \longrightarrow U^{*} \longrightarrow J^{*} \longrightarrow I^{*} \longrightarrow 0
$$

is the exact sequence

$$
0 \longrightarrow S^{n-1} J^{*} \otimes U^{*} \longrightarrow S^{n} J^{*} \longrightarrow S^{n} I^{*} \longrightarrow 0 .
$$

Taking the long exact sequence in cohomology, we see that $I$ and $J$ are equivalent whenever the following condition holds:

$$
H^{i}\left(G / B, S^{n} J^{*} \otimes U^{*}\right)=0 \text { for all } n \geq 0 \text {, for } i \in\{0,1\} .
$$

There is an easy (but very useful) case when (2) is sure to hold; it is due to Demazure [6]. For $\alpha \in \Pi$, let $P_{\alpha}$ denote the minimal parabolic containing $B$ corresponding to $\alpha$. Let $\mathfrak{g}_{\alpha}$ denote the root space in $\mathfrak{g}$ corresponding to the root $\alpha \in \Phi$. 
Proposition 2.2. ([2]) Let $I \subsetneq J$ be two ideals of $\mathfrak{n}$ with $J=I+\mathfrak{g}_{\beta}$ where $\beta \in \Phi^{+}$. If $J$ extends to a representation of $P_{\alpha}$ for some $\alpha \in \Pi$ such that

$$
\left\langle\beta, \alpha^{\vee}\right\rangle=-1
$$

then

$$
H^{i}\left(G / B, S^{n} J^{*} \otimes U^{*}\right)=0 \text { for all } i, n \geq 0 .
$$

In particular, $I$ and $J$ are equivalent.

If two ideals satisfy the hypotheses of Proposition 2.2, we say that they are equivalent via the basic move (with respect to $\alpha$ if we want to emphasize the simple root). Note that in the above proposition the simple roots $\Pi$ are determined by $B$ (and not the opposite Borel as is also common). The basic move already shows up in DeConcini-Lusztig-Procesi's work [5, Section 2.8] and in Mizuno's [13, Lemma 1].

\section{$\S 3 . \quad$ Main properties}

Let $I$ be a $B$-stable ideal in $\mathfrak{n}$. There is a natural map (the moment map)

$$
\pi: G \times{ }^{B} I \longrightarrow \mathfrak{g}
$$

which send $(g, X) \in G \times{ }^{B} I$ to $g . X$.

The image is irreducible (since $I$ is irreducible and $G$ is connected) and it is closed since $G / B$ is projective. The image, which consists of nilpotent elements and is $G$-stable, is thus a union of nilpotent orbits. Since there are only finitely many such orbits in $\mathfrak{g}$, the image is the closure of a single nilpotent orbit $\mathcal{O}_{I}$. We call $\mathcal{O}_{I}$ the associated orbit of $I$; we call its closure $\overline{\mathcal{O}}_{I}$ the $G$-saturation of $I$ and denote it also by G.I. We note that $\mathcal{O}_{I}$ is also the unique nilpotent orbit such that $\mathcal{O}_{I} \cap I$ is dense in $I$.

The fibers of $\pi$ over points in $\mathcal{O}_{I}$ were studied in [5] (in a slightly modified setting) in order to show that the cohomology of a Springer fiber has no odd cohomology. For our purposes we recall the salient facts. First note that $G$ acts on the left on $G \times{ }^{B} I$ and on $\mathfrak{g}$ and the map $\pi$ is clearly equivariant for these actions. For $e \in \mathcal{O}_{I}$, we can study the action of $G_{e}$ on the fiber of $\pi$ over $e$, where $G_{e}$ is the centralizer of $e$ in $G$ under the adjoint action. Clearly, $G_{e}$ preserves the fiber and its identity component $G_{e}^{0}$ preserves each irreducible component of the fiber.

Using the fact that $\mathcal{O}_{I} \cap I$ is open in $I$, one deduces that $\mathcal{O}_{I} \cap I$ is both smooth and irreducible. Consider the subset $G_{I}$ of $G$ defined by

$$
G_{I}:=\left\{g \in G \mid g^{-1} . e \in I\right\} .
$$


As in [5], $G_{I}$ is stable under the action of $B$ on the right and of $G_{e}$ on the left. On the one hand, $G_{e} \backslash G_{I}$ identifies with $\mathcal{O}_{I} \cap I$ and on the other hand, the fiber $\pi^{-1}(e)$ identifies with the image of $G_{I}$ in $G / B$. Then the arguments in [5, Lemma 2.2] go through: the smoothness of $\mathcal{O}_{I} \cap I$ ensures that $\pi^{-1}(e)$ is smooth, and the irreducibility of $\mathcal{O}_{I} \cap I$ ensures that $G_{e}$ acts transitively on the connected components of $\pi^{-1}(e)$ (which are necessarily irreducible, since they are smooth).

Then as mentioned in the introduction and following [5], it makes sense to consider the stabilizer $H_{I}$ in $G_{e}$ of an irreducible component of the fiber. Since $H_{I}$ contains $G_{e}^{0}$, it defines a subgroup

$$
K_{I}:=H_{I} / G_{e}^{0}
$$

of $A(e)$. Both $H_{I}$ and $K_{I}$ are well-defined up to conjugacy in $G_{e}$ and $A(e)$, respectively, since $G_{e}$ permutes the components of the fiber over $e$. Henceforth we make no distinction between $H_{I}$ and $K_{I}$ and other subgroups conjugate to them in $G_{e}$ and $A(e)$, respectively. They are then well-defined independent of the base point $e \in \mathcal{O}_{I}$.

If a parabolic subgroup $P$ stabilizes $I$ with $B \subset P$, then we can form the appropriate moment map $\pi_{P}: G \times^{P} I \rightarrow G . I$. For $e \in \mathcal{O}_{I}$, the fiber $\pi^{-1}(e)$ is a $P / B$-bundle over $\pi_{P}^{-1}(e)$. Hence the subgroup $K_{I}$ will be the same regardless of whether we use $P$ or $B$. This can be useful when trying to compute $K_{I}$ explicitly.

We next consider the perspective of the Stein factorization of $\pi$ :

$$
G \times{ }^{B} I \stackrel{\mu_{1}}{\longrightarrow} Y_{I} \stackrel{\mu_{2}}{\longrightarrow} \overline{\mathcal{O}}_{I} .
$$

The first map $\mu_{1}$ is projective and has connected fibers. The second map $\mu_{2}$ is finite. Both maps are equivariant for $G$. Since $\overline{\mathcal{O}}_{I}$ is affine, so is $Y_{I}$. Moreover, by construction there is an isomorphism of (finitely generated, reduced) $\mathbf{C}$-algebras $\mathbf{C}\left[Y_{I}\right] \cong \mathbf{C}\left[G \times{ }^{B} I\right]$ of the global regular functions of each variety. Since $G \times{ }^{B} I$ is smooth, $Y_{I}$ is a normal variety.

Let us set $\dot{Y}_{I}:=\mu_{2}^{-1}\left(\mathcal{O}_{I}\right)$. Clearly this is a $G$-variety and since $G_{e}$ acts transitively on the components of $\pi^{-1}(e)$, it follows that it also does on the fiber of $\mu_{2}^{-1}(e)$ as the latter is a finite set of points. Hence $G$ acts transitively on $\dot{Y}_{I}$. Furthermore the stabilizer of a point in $\dot{Y}_{I}$ sitting above $e$ identifies with the subgroup $H_{I}$ in $G_{e}$. It follows that

$$
\dot{Y}_{I} \simeq G / H_{I}
$$


Now since $\overline{\mathcal{O}}_{I}-\mathcal{O}_{I}$ has codimension two in $\overline{\mathcal{O}}_{I}$ and $\mu_{2}$ is finite, the complement of $\dot{Y}_{I}$ in $Y_{I}$ has codimension two in $Y_{I}$. Then using that $\dot{Y}_{I}$ is smooth and $Y_{I}$ is normal, it follows that the inclusion of $\dot{Y}_{I}$ into $Y_{I}$ induces a $G$-equivariant isomorphism

$$
\mathbf{C}\left[\dot{Y}_{I}\right] \simeq \mathbf{C}\left[Y_{I}\right]
$$

(see [7]).

We can now prove

Lemma 3.1. Let $I \subseteq J$ be ideals. Then

$$
H^{0}\left(G / B, S^{n} I^{*}\right) \simeq H^{0}\left(G / B, S^{n} J^{*}\right)
$$

for all $n \geq 0$ if and only if $\mathcal{O}_{I}=\mathcal{O}_{J}$ and $K_{I}=K_{J}$.

Proof. The $\mathbf{C}^{*}$-action on $I$ puts a grading on the the regular functions $\mathbf{C}\left[G \times{ }^{B} I\right]$ and then the functions of degree $n$ on $\mathbf{C}\left[G \times{ }^{B} I\right]$ identify as a $G$-module with $H^{0}\left(G / B, S^{n} I^{*}\right)$ (see [7]). Furthermore the inclusion of $G \times{ }^{B} I$ into $G \times{ }^{B} J$ induces a graded $G$-equivariant map of $\mathbf{C}$-algebras

$$
\mathbf{C}\left[G \times{ }^{B} J\right] \longrightarrow \mathbf{C}\left[G \times{ }^{B} I\right]
$$

compatible with the maps of $G$-modules $H^{0}\left(G / B, S^{n} J^{*}\right) \rightarrow H^{0}\left(G / B, S^{n} I^{*}\right)$ for each $n$.

The following diagram commutes (all maps are $G$-equivariant):

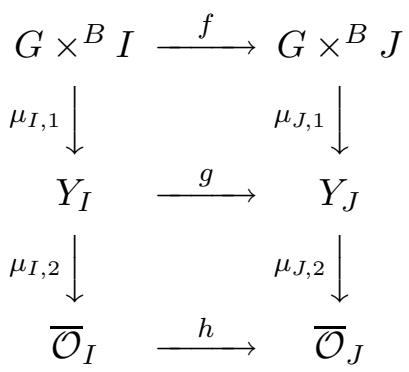

On the level of functions the following diagram commutes:

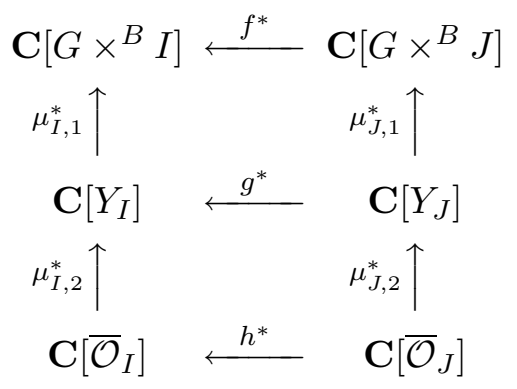


The maps $\mu_{I, 1}^{*}$ and $\mu_{J, 1}^{*}$ are isomorphisms. The map $h^{*}$ is a surjection since $h$ is an injective map between affine varieties. Also, $\mu_{I, 2}^{*}$ and $\mu_{J, 2}^{*}$ are injections since $\mu_{I, 2}$ and $\mu_{J, 2}$ are surjective.

Now if $H^{0}\left(G / B, S^{n} J^{*}\right) \rightarrow H^{0}\left(G / B, S^{n} I^{*}\right)$ is an isomorphism for all $n \geq 0$, this implies that $f^{*}$ is an isomorphism and thus $g^{*}$ and $h^{*}$ are isomorphisms. Hence $\mathcal{O}_{I}=\mathcal{O}_{J}$. But also $Y_{I} \simeq Y_{J}$ as $G$-varieties, whence their open orbits are isomorphic $\dot{Y}_{I} \simeq \dot{Y}_{J}$. Consequently, picking a fixed base point in $e \in \mathcal{O}_{I}$, we see that $H_{I}$ is conjugate in $G_{e}$ to $H_{J}$, whence $K_{I}$ and $K_{J}$ are conjugate in $A(e)$.

For the reverse direction, if $\overline{\mathcal{O}}_{I}=\overline{\mathcal{O}}_{J}$, then $h$ is an isomorphism. Then $g$ must be surjective and it maps $\dot{Y}_{I}$ onto $\dot{Y}_{J}$. This induces a surjection of $G / H_{I}$ to $G / H_{J}$, which shows that some conjugate of $K_{I}$ is contained in $K_{J}$. In particular, if the groups are isomorphic, we have that $\dot{Y}_{I} \simeq \dot{Y}_{J}$. Thus $\mathbf{C}\left[\dot{Y}_{I}\right] \simeq \mathbf{C}\left[\dot{Y}_{J}\right]$ and also $\mathbf{C}\left[Y_{I}\right] \simeq \mathbf{C}\left[Y_{J}\right]$ by (3). Hence $f^{*}$ is an isomorphism and the result follows.

Corollary 3.2. If $I \subseteq J$ and $\mathcal{O}_{I}=\mathcal{O}_{J}$, then $K_{I} \subseteq K_{J}$ for some representatives of these conjugacy classes of subgroups. If $I \sim J$, then $\mathcal{O}_{I}=\mathcal{O}_{J}$ and $K_{I}=K_{J}$.

Proof. The first statement follows from the proof itself. The second statement is clear.

It follows that the basic move of Proposition 2.2 preserves both $\mathcal{O}_{I}$ and $K_{I}$. Compare this proof with the one in [5, Lemma 2.11].

\section{§4. Partial order}

There is a partial order on the set of all $B$-stable ideals of $\mathfrak{n}$ given by inclusion. We now show that this partial order descends to equivalence classes of ideals.

Proposition 4.1. Suppose $I_{1} \sim I_{2}$ and $J_{1} \sim J_{2}$. If $J_{1} \subseteq I_{1}$ and $I_{2} \subseteq J_{2}$, then all four ideals are equivalent.

Proof. There is an injection of $\overline{\mathcal{O}}_{J_{1}}$ into $\overline{\mathcal{O}}_{I_{1}}$ and of $\overline{\mathcal{O}}_{I_{2}}$ into $\overline{\mathcal{O}}_{J_{2}}$. Hence by the second statement of Corollary 3.2 all ideals have the same saturation. Then the first statement of Corollary 3.2 gives that $K_{J_{1}} \subseteq K_{I_{1}}$ and $K_{I_{2}} \subseteq K_{J_{2}}$ and hence that all groups are isomorphic. It follows that $J_{1} \sim I_{1}$ by Lemma 3.1 as $J_{1} \subseteq I_{1}$. 
Hence there is a well-defined partial order on equivalence classes induced by the inclusion of one ideal in another (that the transitive property goes through is an easy modification of the proposition).

Let us denote the equivalence class containing $I$ by $[I]$ and write $[I] \preceq$ $[J]$ to denote when two elements are comparable in the partial order.

It is clear that if $[I] \preceq[J]$ then $\mathcal{O}_{I} \subseteq \overline{\mathcal{O}}_{J}$. We now show that a converse is true: namely if $\mathcal{O}_{1} \subseteq \overline{\mathcal{O}}_{2}$, then there exists ideals $I_{1}, I_{2}$ with $\mathcal{O}_{I_{i}}=\mathcal{O}_{i}$ and $I_{1} \subseteq I_{2}$. In other words the closure order on nilpotent orbits is computable by studying the inclusion order on ideals (where in this case only the saturation of the ideal is needed). As mentioned in the introduction, this was shown by Mizuno in the exceptional groups in a case-by-case fashion.

By the Jacobson-Morozov theorem every nilpotent element $e$ can be embedded in an $\mathfrak{s l}_{2}$-triple $\{e, h, f\}$ in $\mathfrak{g}$. Let $\mathfrak{g}_{h, i}$ be the $i$-eigenspace for $\operatorname{ad}(h)$, written as $\mathfrak{g}_{i}$ when there is no confusion about the semisimple element $h$. Let $\mathfrak{g}_{h, \geq i}$ and $\mathfrak{g}_{h,>i}$ denote the obvious sum of eigenspaces. Then if $h \in \mathfrak{h}$ and $\alpha(h) \geq 0$ for all $\alpha \in \Pi$ (that is, $h$ is dominant), then

$$
I=\mathfrak{g}_{\geq 2}
$$

is a $B$-stable ideal of $\mathfrak{n}$. Moreover, $\mathcal{O}_{I}=\mathcal{O}_{e}$, the orbit through $e$, see [3]. We call $I$ the Dynkin ideal for $\mathcal{O}_{e}$.

THEOREM 4.2. The closure order on nilpotent orbits is obtained from the partial order on equivalence classes of ideals by passing to the saturation of an ideal.

Proof. Suppose that $\mathcal{O}_{1} \subseteq \overline{\mathcal{O}}_{2}$ where $\mathcal{O}_{1} \neq \mathcal{O}_{2}$. We would like to find ideals $I \subset J$ with $\mathcal{O}_{I}=\mathcal{O}_{1}$ and $\mathcal{O}_{J}=\mathcal{O}_{2}$. It suffices to consider the case when there is no orbit in between $\mathcal{O}_{1}$ and $\mathcal{O}_{2}$ in the closure ordering since any descending chain of closed subvarieties of $\mathfrak{g}$ must be finite.

Let $J=\mathfrak{g}_{\geq 2}$ be the Dynkin ideal for the orbit $\mathcal{O}_{2}$ with respect to a dominant semisimple element $h^{\prime} \in \mathfrak{h}$. For this proof we omit the subscript $h^{\prime}$.

Now $\mathcal{O}_{1} \cap J \neq \emptyset$ since $\mathcal{O}_{1} \subset G$.J. Pick $e \in \mathcal{O}_{1} \cap J$ and write $e=x+y$ where $x \in \mathfrak{g}_{2}$ and $y \in \mathfrak{g}_{>2}$. Note that $x \notin \mathcal{O}_{2}$ since

$$
\mathcal{O}_{2} \cap \mathfrak{g}_{\geq 2}=\left(\mathcal{O}_{2} \cap \mathfrak{g}_{2}\right)+\mathfrak{g}_{>2}
$$

(see [4, Lemma 4.14]). 
Let $L \subset G$ be the subgroup with Lie algebra $\mathfrak{g}_{0}$. By Kawanaka's classification of $L$ orbits on $\mathfrak{g}_{2}$ [9], there exists $h \in \mathfrak{h}$ such that $\{\tilde{x}, h, f\}$ forms an $\mathfrak{s l}_{2}$-triple for some $\tilde{x} \in L . x$ and furthermore that

$$
G . \tilde{x} \cap\left(\mathfrak{g}_{h, \geq 2} \cap \mathfrak{g}_{2}\right)
$$

is dense in $\mathfrak{g}_{h, \geq 2} \cap \mathfrak{g}_{2}$.

Now set

$$
I:=\left(\mathfrak{g}_{h, \geq 2} \cap \mathfrak{g}_{2}\right)+\mathfrak{g}_{>2} .
$$

Then $I \subset \mathfrak{n}$ and we wish to show that $I$ is $B$-stable. Appealing to [9] again, we can choose $h$ so that $h$ is dominant for the simple roots $\Pi_{L}$ which come from $L$. Assume that $\alpha \in \Pi_{L}$. Then $\alpha\left(h^{\prime}\right)=0$ and so $\operatorname{ad}\left(\mathfrak{g}_{\alpha}\right)$ preserves each $\mathfrak{g}_{i}$. Furthermore $\alpha(h) \geq 0$ and so ad $\left(\mathfrak{g}_{\alpha}\right)$ preserves $\mathfrak{g}_{h, \geq 2}$. Consequently, $\operatorname{ad}\left(\mathfrak{g}_{\alpha}\right)(I) \subseteq I$. On the other hand, if $\alpha \in \Pi \backslash \Pi_{L}$, then $\alpha\left(h^{\prime}\right)>0$ and thus $\operatorname{ad}\left(\mathfrak{g}_{\alpha}\right)$ maps $\mathfrak{g}_{\geq 2}$ into $\mathfrak{g}_{>2}$. Consequently, $\operatorname{ad}\left(\mathfrak{g}_{\alpha}\right)(I) \subseteq I$ in this case too. Thus $I$ is an $\operatorname{ad}(\mathfrak{b})$-stable ideal and so also $B$-stable.

To finish the proof, note that $I$ does not intersect $\mathcal{O}_{2}$. Indeed if it did, then (4) would imply that $\mathcal{O}_{2} \cap\left(\mathfrak{g}_{h, \geq 2} \cap \mathfrak{g}_{2}\right)$ is non-empty and hence it is dense in $\mathfrak{g}_{h, \geq 2} \cap \mathfrak{g}_{2}$. This would contradict the fact that $\tilde{x} \notin \mathcal{O}_{2}$. On the other hand some $L$ - conjugate of $e$ lies in $I$, so $I$ does intersect $\mathcal{O}_{1}$. Also $I \subset J$. It follows that

$$
\mathcal{O}_{1} \subseteq G . I \subsetneq G . J
$$

and hence that $\overline{\mathcal{O}}_{1}=$ G.I since by assumption there is no orbit between $\mathcal{O}_{1}$ and $\mathcal{O}_{2}$.

\section{§5. Computing the $\mathcal{O}_{I}$ and $K_{I}$}

In this section we gather some results about ideals that we can use to compute $\mathcal{O}_{I}$ and $K_{I}$ for an ideal $I$. These allow us to determine the equivalence classes in the exceptional groups and thus compute the partial order.

\subsection{Ideals of minimal dimension}

One main question which arises is to find, given a nilpotent orbit $\mathcal{O}$, the minimal dimension of an ideal $I$ such that $G . I=\overline{\mathcal{O}}$. More generally, if we also make some assumption on $B_{e}$ for $e \in \mathcal{O} \cap I$, we would like to know the minimal dimension of such an $I$. This will be helpful to show that $K_{I}$ does not intersect a given conjugacy class in $A(e)$.

Here is our main result about the lower bound on the dimension of $I$. The case where $s \in B_{e}^{0}$ was known to Kawanaka [8]. 
Proposition 5.1. Let $\mathcal{O}$ be a nilpotent orbit. Let $I$ be an ideal and $e \in \mathcal{O} \cap I$ where $\mathcal{O}_{I}=\mathcal{O}$. Let $s \in B_{e}$ be a semisimple element. Then

$$
\operatorname{dim} I \geq \operatorname{dim} B-\operatorname{dim}\left(B_{G_{e}}\right)+\operatorname{rank}\left(G_{e}\right)-\operatorname{rank}\left(G_{s, e}\right)
$$

where $B_{G_{e}}$ is a Borel subgroup of $G_{e}$ and $G_{s, e}$ denotes the elements which commute with $s$ and also commute with e under the adjoint action.

Proof. First

$$
\operatorname{dim} I \geq \operatorname{dim} B-\operatorname{dim} B_{e}
$$

Next, there exists a maximal torus $S$ of $B_{e}$ which is invariant under conjugation by $s$ (see [22]). In other words $s \in N_{B}(S)$, the normalizer of $S$ in $B$. However, $B$ is connected and solvable and $S$ consists of semisimple elements. Thus $Z_{B}(S)=N_{B}(S)$ by [21, Corollary 6.3.6]. It follows that $S \subset G_{s, e}$ and so

$$
\operatorname{rank}\left(B_{e}\right) \leq \operatorname{rank}\left(G_{s, e}\right) .
$$

This together with the fact that $B_{e}$ is solvable and so $B_{e} \subseteq B_{G_{e}}$ gives the inequality

$$
\operatorname{dim} B_{e} \leq \operatorname{dim}\left(B_{G_{e}}\right)-\left(\operatorname{rank}\left(G_{e}\right)-\operatorname{rank}\left(G_{s, e}\right)\right)
$$

and the result follows.

We can easily obtain the information to compute the lower bound of the proposition. The rank of $G_{e}$ equals the rank of $G$ less the subscript in the Bala-Carter designation of $\mathcal{O}$ (which denotes the rank of the minimal Levi subalgebra containing $e$ ). If we insist that $s$ lies in a specified conjugacy class $C$ in $A(e)$, then the rank of $G_{s, e}$ is the rank of $G$ less the subscript in the generalized Bala-Carter designation of $C$ in [16]. In other words, the difference of ranks in the proposition is the difference of subscripts of the two Bala-Carter designations. Let $N_{e}$ denote the number of positive roots in the reductive part of $G_{e}$. Given the weighted Dynkin diagram for $\mathcal{O}$, we can compute the dimensions of $\mathfrak{g}_{i}$. Then we have

$$
\begin{aligned}
\operatorname{dim} B_{G_{e}} & =\operatorname{dim} G_{e}-N_{e} \\
& =\operatorname{dim} \mathfrak{g}_{0}+\operatorname{dim} \mathfrak{g}_{1}-\frac{1}{2}\left[\operatorname{dim}\left(\mathfrak{g}_{0}\right)-\operatorname{dim}\left(\mathfrak{g}_{2}\right)-\operatorname{rank}\left(G_{e}\right)\right] \\
& =\operatorname{dim} \mathfrak{g}_{1}+\frac{1}{2}\left[\operatorname{dim}\left(\mathfrak{g}_{0}\right)+\operatorname{dim}\left(\mathfrak{g}_{2}\right)+\operatorname{rank}\left(G_{e}\right)\right]
\end{aligned}
$$


Of course, in practice we can also just read this information from tables in $[3]$.

Let us set

$$
m_{\mathcal{O}}=\operatorname{dim} B-\operatorname{dim}\left(B_{G_{e}}\right)
$$

for $e \in \mathcal{O}$. More generally for $e \in \mathcal{O}$ and $C$ a conjugacy class in $A(e)$, let $s \in G_{e}$ be a semisimple element representing the class $C$ and set

$$
m_{\mathcal{O}, C}=\operatorname{dim} B-\operatorname{dim}\left(B_{G_{e}}\right)+\operatorname{rank}\left(G_{e}\right)-\operatorname{rank}\left(G_{s, e}\right) .
$$

These values are well-defined independent of the choice of $e$ or $s$.

EXAMPLE 5.2. As an example, consider an element $e$ in the orbit of type $D_{4}\left(a_{1}\right)$ in $E_{6}$. Then $\operatorname{rank}\left(G_{e}\right)=6-4=2$ and $\operatorname{dim} \mathfrak{g}_{0}=20$ and $\operatorname{dim} \mathfrak{g}_{2}=18$, so that $\operatorname{dim} B_{G_{e}}=20$. For $E_{6}, \operatorname{dim} B=42$ and thus the minimal dimension for an $I$ with $\mathcal{O}_{I}=\mathcal{O}$ is $m_{\mathcal{O}}=22$. There does exist an ideal achieving this value (see Section 5.2).

Now if we specify that the image of $s \in B_{e}$ has order two in $A(e)$, then the Bala-Carter designation of the pair $(e, s)$ is $A_{3}+2 A_{1}$, so that $\operatorname{rank}\left(G_{e}\right)-\operatorname{rank}\left(G_{s, e}\right)=1$ and the minimal dimension of such an $I$ is 23 . There does exist an ideal with these properties.

Next if we specify that the image of $s$ has order three, then the minimal possible dimension of such an $I$ is 24 . Mysteriously there does not exist any ideal satisfying these properties. The smallest such ideal has dimension 25.

We now consider what happens when $\operatorname{dim} I=m_{\mathcal{O}_{I}}$.

Corollary 5.3. Suppose that $I$ is an ideal with $\operatorname{dim} I=m_{\mathcal{O}_{I}}$. Then

(1) [8] $B$ acts transitively on $\mathcal{O}_{I} \cap I$.

(2) The image of $B_{e}$ in $A(e)$ equals $K_{I}$ for any $e \in \mathcal{O}_{I} \cap I$.

Proof. Let $e \in \mathcal{O}_{I} \cap I$. Since $\operatorname{dim} I=m_{\mathcal{O}_{I}}$, the proof of Proposition 5.1 shows that $B_{e}^{0}=B_{G_{e}}$ and $\operatorname{dim} I=\operatorname{dim} B-\operatorname{dim} B_{e}$. Hence the $B$-orbit through $e$ is dense. Since this holds for any point in $\mathcal{O}_{I} \cap I$, which is irreducible, $B$ acts transitively on $\mathcal{O}_{I} \cap I$. From this we can deduce that $G_{e}$ acts transitively on the fiber $X=\pi^{-1}(e)$. Indeed a typical element in the fiber is $\left(g, e^{\prime}\right)$ where $g . e^{\prime}=e$ and $e^{\prime} \in I$. The $B$-transitivity gives that $\left(g, e^{\prime}\right)=\left(g b^{-1}, b . e^{\prime}\right)=\left(g b^{-1}, e\right)$ for some $b \in B$. Hence $\left(g, e^{\prime}\right)=g b^{-1} \cdot(1, e)$ and $g b^{-1} \in G_{e}$. Incidentally this also shows that each component of $X$ is isomorphic to the flag variety of $G_{e}^{0}$. 
To complete the proof, let $s \in H_{I}$ where $H_{I}$ is the stabilizer in $G_{e}$ of the irreducible component of $X$ containing $(1, e)$. Then by definition this means that $s .(1, e)$ lies in the same irreducible component of $X$ as $(1, e)$. Since $G_{e}^{0}$ acts transitively on each component of $X$, we have $(s, e)=(g, e)$ for some $g \in G_{e}^{0}$, where the equality is in $G \times{ }^{B} I$. In other words, $s b^{-1}=g$ and $b . e=e$ for some $b \in B$. But then $b \in B_{e}$ and $g^{-1} s=b$ and thus $b$ and $s$ have the same image in $A(e)$, proving that the image of $H_{I}$ in $A(e)$ coincides with the image of $B_{e}$.

Usually the corollary is enough to determine $K_{I}$ when $\operatorname{dim} I=m_{\mathcal{O}_{I}}$ as we discuss in Section 5.3. Of course it would be helpful to know that this minimum value is actually achieved. This is the case in the exceptional groups, which was shown by Kawanaka [8] in his work on Gelfand-Graev representations. Kawanaka's observation relies on Mizuno's calculations (which in a sense we are trying to simplify and make more uniform in this paper). In the next subsection we give a conjectural algorithm which produces an ideal of minimal dimension (re-verifying Kawanaka's observation). It seems reasonable to conjecture for all groups that

ConjeCture 5.4. For each orbit $\mathcal{O}$, there exists an ideal $I$ with $\mathcal{O}_{I}=$ $\mathcal{O}$ and $\operatorname{dim} I=m_{\mathcal{O}}$.

The following is also going to be useful to compute $K_{I}$.

Corollary 5.5. Let $I$ be an ideal with $\operatorname{dim} I=m_{\mathcal{O}_{I}}+1$. Let $e \in \mathcal{O}_{I} \cap I$ and assume that there is a semisimple element $s \in B_{e}$ with $\operatorname{rank}\left(G_{e}\right)-$ $\operatorname{rank}\left(G_{s, e}\right)=1$. Suppose also that there exists a parabolic subgroup $P=P_{\alpha}$ of semisimple rank one that stabilizes $I$. Then the image of $P_{e}$ in $A(e)$ is exactly $K_{I}$.

Proof. By Proposition 5.1 and the assumption on $s$,

$$
\operatorname{dim} I \geq \operatorname{dim} P-\operatorname{dim} P_{e} \geq \operatorname{dim} B-\operatorname{dim} B_{e}>\operatorname{dim} B-\operatorname{dim} B_{G_{e}} .
$$

The hypothesis on the dimension of $I$ then shows that $\operatorname{dim} I=\operatorname{dim} P-$ $\operatorname{dim} P_{e}$. Let $X_{P}$ be the fiber of the bundle $G \times{ }^{P} I$ over $e$. Then $\operatorname{dim} X_{P}=$ $\operatorname{dim} G_{e}-\operatorname{dim} P_{e}$.

Let $Z$ be the orbit of $G_{e}^{0}$ through $(1, e)$ in $X_{P}$. Then $\operatorname{dim} Z=\operatorname{dim} G_{e}-$ $\operatorname{dim} P_{e}=\operatorname{dim} X_{P}$, so $Z$ is dense in its irreducible component of $X_{P}$. Let $s \in H_{I}$ be the stabilizer in $G_{e}$ of this component. Then $s . Z \cap Z$ must be 
non-empty. Hence for some $g, h \in G_{e}^{0}, s \cdot(g, e)=(h, e)$ in $G \times^{P} I$. Thus for some $p \in P$ we have $s g p^{-1}=h$ and $p . e=e$. This means that $p \in P_{e}$ and $p$ and $s$ have the same image in $A(e)$.

Finally we note that if an ideal $I$ is stabilized by a parabolic subgroup $P$ such that $G \times{ }^{P} I$ has the same dimension as $\mathcal{O}_{I}$, then the fiber over $e \in \mathcal{O}_{I}$ being of dimension zero also ensures that the image of $P_{e}$ is $K_{I}$. This is useful when an ideal is induced from a Dynkin ideal in a Levi subalgebra since this dimension property always holds in that case.

\subsection{Importing lower rank ideals}

We now describe a method to find the saturation of ideals in $\mathfrak{g}$ and get some structure on $K_{I}$ from the knowledge of the saturation of ideals in certain subalgebras of $\mathfrak{g}$.

Let $\tilde{\Pi}=\Pi \cup\{-\theta\}$ be the extended simple roots of $\mathfrak{g}$, where $\theta \in \Phi^{+}$is the highest root with respect to $\Pi$. For $J \subsetneq \tilde{\Pi}$ define $\Phi_{J}$ to be the roots of $\Phi$ which are integral combinations of the roots in $J$. Let

$$
\mathfrak{g}_{J}:=\mathfrak{h} \oplus \bigoplus_{\alpha \in \Phi_{J}} \mathfrak{g}_{\alpha} .
$$

Let $G_{J}$ be the corresponding group in $G$. Then we call $\mathfrak{g}_{J}$ a standard pseudo-Levi subalgebra of $\mathfrak{g}$.

Now suppose $e \in \mathfrak{l}=\mathfrak{g}_{J}$ is a nilpotent element for some pseudo-Levi subalgebra. Let $h \in \mathfrak{h} \cap[\mathfrak{l}, \mathfrak{l}]$ be a Dynkin element for $e$ in $\mathfrak{l}$. Then $\mathfrak{l}_{h, \geq 2}$ defines an ideal for $\mathfrak{l}$ for some Borel subalgebra $\mathfrak{b}_{\mathfrak{l}}$ of $\mathfrak{l}$ containing $\mathfrak{h}$ and contained in the parabolic subalgebra $\mathfrak{l}_{h, \geq 0}$. Then the intersection $\mathcal{O}_{e} \cap \mathfrak{l}_{h, \geq 2}$ is dense in $\mathfrak{l}_{h, \geq 2}$. In other words

$$
G_{J} \cdot \mathfrak{l}_{h, \geq 2}=\overline{G_{J} \cdot e}
$$

in $\mathfrak{l}$.

Let $I_{\mathfrak{l}} \subset \mathfrak{l}_{h, \geq 2}$ be an ideal with respect to $\mathfrak{b}_{\mathfrak{l}}$ whose saturation is also $\overline{G_{J} . e}$. Let $w \in W$ be an element such that $w(h)$ is dominant for $\mathfrak{g}$. Certainly

$$
w\left(I_{\mathfrak{l}}\right) \subset \mathfrak{g}_{w(h), \geq 2} .
$$

Since $\mathcal{O}_{e} \cap I_{\mathfrak{l}}$ is dense in $I_{\mathfrak{l}}$, the same holds for $w\left(I_{\mathfrak{l}}\right)$. Also, $\mathcal{O}_{e} \cap \mathfrak{g}_{w(h), \geq 2}$ is dense in $\mathfrak{g}_{w(h), \geq 2}$. Hence the $B$-stable ideal $I$ generated by $w\left(I_{\mathfrak{l}}\right)$ (meaning the smallest ideal containing the latter) must also have this property. In other words, $I$ has saturation equal to $\overline{\mathcal{O}}_{e}$. In this way we have located an ideal $I$ contained in $\mathfrak{g}_{w(h), \geq 2}$ with the same saturation. 
Moreover if the pair $(\mathfrak{l}, e)$ corresponds to the conjugacy class $C \subset A(e)$ as in [16], then there exists $s \in T$ such that $s \in G_{e}$ and the image of $s$ in $A(e)$ lies in $C$. Consequently, $w(s)$ lies in $G_{w . e}$ and this shows that the image of $B_{w . e}$ in $A(w . e)$ intersects the conjugacy class $w(C)$. This is all well-defined since the component groups $A(e)$ and $A(g . e)$ are isomorphic and any isomorphism between them coincides on conjugacy classes. Hence we can conclude that $K_{I}$ intersects the conjugacy class $C$ specified by the pair $(\mathfrak{l}, e)$.

For purposes of computation it is easiest to specify an ideal in $\mathfrak{n}$ by its minimal roots. Hence we are led to find the minimal roots for $I$, the ideal generated by $w\left(I_{\mathfrak{l}}\right)$. We can not expect that they are always $w$ applied to the minimal roots of $I_{\mathfrak{l}}$ in $\mathfrak{l}$ since for one thing the minimal roots of $I$ must be $W$-conjugate to a subset of $\Pi[19]$. However under some additional assumptions the situation is as good as can be expected.

Let us assume that the simple roots of $\mathfrak{l}$ are determined by $\mathfrak{b}$, so that the simple roots of $\mathfrak{l}$ are $J \cap \Pi$ together with possibly one additional positive root $\gamma$. In other words, $\mathfrak{b}_{\mathfrak{l}}=\mathfrak{b} \cap \mathfrak{l}$. Also assume that the element $h \in \mathfrak{h}$ has the property that $\alpha(h) \in\{0,2\}$ for all $\alpha \in J \cap \Pi$ and that $\gamma(h)=2$ if $\gamma$ exists. Let $l(-)$ denote the length function of $W$ defined with respect to the simple reflections $\left\{s_{\alpha} \mid \alpha \in \Pi\right\}$.

Proposition 5.6. Under the assumptions of the previous paragraph, let $\beta_{1}, \ldots, \beta_{m}$ be the minimal roots of $I_{\mathfrak{l}}$. Assume that $\beta_{i}(h)=2$ for all $\beta_{i}$ and that $\beta_{1}=\gamma$ if $\gamma$ exists. Let $w \in W$ be the element of minimal length such that $w(h)$ is dominant. Then the minimal roots of the ideal I generated in $\mathfrak{n}$ by $w\left(I_{\mathfrak{l}}\right)$ are

$$
w\left(\beta_{2}\right), \ldots, w\left(\beta_{m}\right)
$$

and possibly also $w(\gamma)$. In particular if $J \subset \Pi$, then the minimal roots are exactly $\left\{w\left(\beta_{i}\right)\right\}$.

Proof. If $\alpha(h)=2$, then $w(\alpha)$ is a positive root since $w(\alpha) \in \mathfrak{g}_{w(h), 2}$ and $w(h)$ is dominant. Also $\gamma(h)=2$ and thus $w(\gamma)$ is a positive root. Now by the next lemma $w(\alpha)$ is a positive root even when $\alpha(h)=0$ for $\alpha \in J \cap \Pi$ (indeed it is a simple root). We conclude that all the simple roots of $\mathfrak{l}$ remain positive roots after applying $w$. Since every root of $w\left(I_{\mathfrak{l}}\right)$ is of the form

$$
w\left(\beta_{j}\right)+\beta
$$

where $\beta$ is a sum (with repetitions) of $w(\gamma)$ and the $w(\alpha)$ where $\alpha \in J \cap \Pi$, this shows that the minimal roots of $I$ are found among the roots $\left\{w\left(\beta_{i}\right)\right\}$. 
It remains to show that for $i, j \neq 1$ that $w\left(\beta_{i}\right)$ and $w\left(\beta_{j}\right)$ are not comparable and also that $w\left(\beta_{i}\right)$ is never less than $w(\gamma)$.

Suppose that

$$
w\left(\beta_{i}\right)-w\left(\beta_{j}\right)=\sum \nu_{k}
$$

is a sum of positive roots. Then $\sum \nu_{k}(w(h))=0$ and since $w(h)$ is dominant, all $\nu_{k}(w(h))=0$. Let $w=s_{r_{1}} \cdots s_{r_{p}}$ be a reduced expression for $w$. Now $\beta_{i}$ and $\beta_{j}$ are both non-comparable positive roots of $\Phi_{J \cap \Pi}$ by assumption and hence they must be non-comparable as roots in $\Phi^{+}$. Thus some $\nu_{k}$ has the property that $w^{-1}\left(\nu_{k}\right)$ is a negative root. It follows that there exists a $q$ such that $s_{r_{q}} \cdots s_{r_{1}}\left(\nu_{k}\right)$ is the simple root $\alpha_{r_{q+1}}$. But then $\alpha_{r_{q+1}}\left(s_{r_{q}} \cdots s_{r_{1}} w(h)\right)=0$, that is,

$$
\left(s_{r_{q+1}} s_{r_{q}} \cdots s_{r_{1}} w\right)(h)=\left(s_{r_{q}} \cdots s_{r_{1}} w\right)(h),
$$

and so we can omit $s_{r_{q+1}}$ from the reduced expression of $w$ and get an element of shorter length which makes $h$ dominant. This is a contradiction.

A similar proof shows that $w(\gamma)$ is never less than $w\left(\beta_{i}\right)$ for $i>1 \mathrm{using}$ the fact that $\beta_{i}-\gamma$ can not be a sum of positive roots of $\Phi^{+}$.

Let $V=Y \otimes_{\mathbb{Z}} \mathbb{Q}$, the vector space spanned by the cocharacters of $G$.

Lemma 5.7. Let $\lambda \in V$. Let $w \in W$ be the element of $W$ of minimal length such that $w(\lambda)$ is dominant. Then $\alpha(\lambda)=0$ for $\alpha \in \Pi$ implies that $w(\alpha) \in \Pi$.

Proof. The fact that $\alpha(\lambda)=0$ implies that $w s_{\alpha}(\lambda)=w(\lambda)$. But then $l\left(w s_{\alpha}\right)>l(w)$ by the minimal length of $w$ and so $w(\alpha) \in \Phi^{+}$since $\alpha$ is simple. Write $w(\alpha)=\sum \gamma_{i}$ where $\gamma_{i} \in \Pi$. Of course $\langle w(\alpha), w(\lambda)\rangle=$ $\langle\alpha, \lambda\rangle=0$. Hence $\sum \gamma_{i}(w(\lambda))=0$. But $w(\lambda)$ is dominant and this forces $\gamma_{i}(w(\lambda))=0$ for all $i$. Then $s_{\gamma_{i}} w(\lambda)=w(\lambda)$ and the minimal length of $w$ implies that $w^{-1}\left(\gamma_{i}\right) \in \Phi^{+}$for all $i$. In other words, $\alpha=\sum w^{-1}\left(\gamma_{i}\right)$ is written as a sum of positive roots. But $\alpha$ is simple so there is only one term in the summation. That is, $w(\alpha)$ is a simple root.

We conjecture that the method of Proposition 5.6 can be used to find an ideal with $\operatorname{dim} I=m_{\mathcal{O}_{I}}$, when $\mathcal{O}_{I}$ is not distinguished.

Conjecture 5.8. Let $\mathcal{O}$ be a nilpotent orbit in $\mathfrak{g}$. Let $\mathfrak{l}$ be a standard minimal Levi subalgebra such that $\mathcal{O} \cap \mathfrak{l}$ is nonempty. Then there exists an ideal $I_{\mathfrak{l}}$ in $\mathfrak{l}$ and $w \in W$ as in Proposition 5.6 such that the ideal I generated by $w\left(I_{\mathfrak{l}}\right)$ satisfies $\operatorname{dim} I=m_{\mathcal{O}_{I}}$. 
We have verified the conjecture in the exceptional groups. Certainly $I_{\mathfrak{l}}$ has to be an ideal of minimal dimension in $\mathfrak{l}$ by Proposition 5.6. Thus the conjecture would reduce the problem of finding ideals with $\operatorname{dim} I=m_{\mathcal{O}_{I}}$ to the case of distinguished orbits. These need to be handled in an ad hoc manner, which we sketch in the next subsection. In fact in the exceptional groups these were basically handled in [5, Section 4.1].

As an example illustrating the proposition and the conjecture, consider the orbit of type $D_{4}\left(a_{1}\right)$ in $E_{6}$.

ExAMPLE 5.9. There are three ideals in a subalgebra $\mathfrak{l}$ of type $D_{4}$ which achieve the minimal value for the orbit of type $D_{4}\left(a_{1}\right)$ in $\mathfrak{l}$. One of them has minimal roots

$$
\left\{\alpha_{2}, \alpha_{3}+\alpha_{4}, \alpha_{6}\right\}
$$

(embedding $D_{4}$ into $E_{6}$ using the notation of [18]). Writing $h \in[\mathfrak{l}, \mathfrak{l}]$ in the basis of simple coroots of $E_{6}$ gives

$$
4 \alpha_{2}^{\vee}+6 \alpha_{3}^{\vee}+4 \alpha_{4}^{\vee}+4 \alpha_{6}^{\vee}
$$

The element $w$ of minimal length such that $w(h)$ is dominant is

$$
s_{3} s_{4} s_{2} s_{6} s_{3} s_{4} s_{2} s_{5} s_{1}
$$

Applying this to $\alpha_{2}, \alpha_{3}+\alpha_{4}, \alpha_{6}$ gives

$$
\alpha_{1}+\alpha_{2}+\alpha_{3}, \alpha_{3}+\alpha_{4}+\alpha_{5}+\alpha_{6}, \alpha_{2}+\alpha_{3}+\alpha_{4},
$$

respectively. Also $w\left(\alpha_{3}\right)=\alpha_{6}$, a simple root as predicted by Lemma 5.7. Proposition 5.6 then guarantees that the ideal in $E_{6}$ with minimal roots $\alpha_{1}+\alpha_{2}+\alpha_{3}, \alpha_{3}+\alpha_{4}+\alpha_{5}+\alpha_{6}, \alpha_{2}+\alpha_{3}+\alpha_{4}$ has saturation equal to the closure of the orbit of type $D_{4}\left(a_{1}\right)$. A computation shows that it has the predicted minimal dimension of 22 .

We finish the subsection by noting a sort of converse to Proposition 5.6. That proposition produces ideals for which the image of $B_{e}$ intersects a fixed conjugacy class in $A(e)$. Conversely, let $I$ be an ideal and let $e \in \mathcal{O}_{I} \cap I$. Suppose that there exists $s \in B_{e}$ whose image in $A(e)$ intersects a fixed conjugacy class $C$. By taking its semisimple part, $s$ may be taken to be semisimple and by conjugating by $B$ we can assume that $s \in T$. Then $\mathfrak{g}_{s} \cap \mathfrak{b}$ is a Borel subalgebra of $\mathfrak{g}_{s}$. It follows that $I \cap \mathfrak{g}_{s}$ is an ideal of $\mathfrak{g}_{s}$ with respect to a Borel subgroup of $G_{s}$. Moreover, since $e \in I \cap \mathfrak{g}_{s}$, we 
have that the saturation of $I \cap \mathfrak{g}_{s}$ is $\overline{G_{s} . e}$. Now by [16] $\mathfrak{g}_{s}$ is conjugate by $W$ to a standard pseudo-Levi subalgebra of $\mathfrak{g}$. Hence if we understand the saturation of ideals in pseudo-Levi subalgebras, we can put a limit on the conjugacy classes which intersect the image of $B_{e}$.

\subsection{Computations in the exceptional groups}

Here is our algorithm to compute the equivalence classes in the exceptional groups. First we compute the equivalence relation generated by the basic move (using a computer). This produces an upper limit on the number of equivalence classes of 6 in $G_{2} ; 25$ in $E_{6} ; 64$ in $E_{7}$; and 128 in $E_{8}$. The output of this operation will be available at www .math . umass .edu/ esommers/partialorder.html.

Next we have to decide which ideals have saturation corresponding to distinguished orbits. We can use the Dynkin ideals $\mathfrak{g}_{\geq 2}$ and Proposition 5.6 for pseudo-Levi subalgebras which are not Levi subalgebras. As a last resort, we can induce a Dynkin ideal from a proper Levi subalgebra l: namely take the minimal roots for a Dynkin ideal in $\mathfrak{l}$ and add to these the simple roots of $\mathfrak{g}$ not in $\mathfrak{l}$ to form an ideal in $\mathfrak{n}$. By [12] the dimension of the saturation of this ideal is known and then inclusion of ideals is enough to determine the actual saturation of the ideal. This is necessary, for example, for the orbit of type $E_{7}\left(a_{5}\right)$ in $E_{7}$.

Then we use the Dynkin ideals $\mathfrak{g}_{\geq 2}$ and the ideals coming from Proposition 5.6 for Levi subalgebras to compute the saturation of ideals in each block of ideals determined by the basic move. It turns out that this is sufficient to determine the saturation of all ideals.

Next we seek to compute $K_{I}$ for each $I$. For an ideal equivalent to $\mathfrak{g}_{\geq 2}$, then $K_{I}=A(e)$. At the other extreme if $I$ satisfies $\operatorname{dim} I=m_{\mathcal{O}_{I}}$, then each conjugacy class of $K_{I}$ is represented by an element $s \in B_{e}$ by Corollary 5.3. This can be chosen to be semisimple and by applying $B$, can be taken to be $s \in T$ as in Section 5.2. From this it follows that $\mathfrak{g}_{s} \cap I$ must be an ideal in $\mathfrak{g}_{s}$ (taking the positive roots to be those in $\mathfrak{b} \cap \mathfrak{g}_{s}$ ). This means that for such ideals, it is purely a combinatorial problem to determine the conjugacy classes of $A(e)$ which intersect $K_{I}$. However, usually we can avoid all work, since it often happens that $\operatorname{rank}\left(G_{e}\right)>\operatorname{rank}\left(G_{s, e}\right)$ and thus by Proposition $5.1, K_{I}$ misses the conjugacy class represented by $s$. In this manner we can determine whether $K_{I}=1$ or $K_{I}=S_{2}$ whenever $A(e)=S_{2}$.

Now when $A(e)=S_{3}$, it turns out in many cases that when $s \in B_{e}$ represents the 3 -cycle that $\operatorname{rank}\left(G_{e}\right)-\operatorname{rank}\left(G_{s, e}\right)=2$ and for $s$ representing the 2 -cycle that $\operatorname{rank}\left(G_{e}\right)-\operatorname{rank}\left(G_{s, e}\right)=1$. This allows us to show that 
$K_{I}$ is trivial for an ideal achieving the minimal value for $\mathcal{O}_{I}$. Next it also turns out that there is an ideal satisfying the hypotheses of Corollary 5.5 with $s \in B_{e}$ representing the 2-cycle (by a use of Proposition 5.6). Hence Proposition 5.1 and Corollary 5.5 give that the 3 -cycle does not show up in $K_{I}$ and hence $K_{I}=S_{2}$. We note that in all of these cases, there is no ideal $I$ with $K_{I}$ containing a 3-cycle which achieves the value $m_{\mathcal{O}_{e}, C}$ for $C$ containing the 3 -cycles. Indeed the minimal achieved is $m_{\mathcal{O}_{e}, C}+1$. We do not have any explanation for this phenomenon.

For the remaining cases (including the distinguished cases), we do need to decide when an ideal coming from a pseudo-Levi subalgebra can be conjugated by $W$ into $I$. We omit the details.

We note that most of these computations can be obtained via Kawanaka's use of Mizuno's detailed computations [8], [13]. Additionally the computations for the distinguished orbits (although not all of the details) are contained in [5].

The equivalence classes are thus computed. The partial order is given in the appendices.

We used these results to double check the partial order on nilpotent orbits in [13], [3], and [20] (by Theorem 4.2). We were able thus to confirm that Spaltenstein's graph of the partial order is totally correct (although there are some redundant lines). In Carter's graphs the following inclusions are missing: in $E_{7}, D_{5}\left(a_{1}\right)+A_{1} \leq D_{6}\left(a_{2}\right)$; in $E_{8}, E_{8}\left(b_{6}\right) \leq E_{6}+A_{1}$ and $A_{3} \leq A_{3}+A_{1}$. There are several errors in Mizuno's graphs.

As noted in the introduction, these calculations also allow the work in [10] and Elashvili's induction tables in [20] to be verified.

\section{$\S 6$. Main conjectures}

We conclude by stating some conjectures about equivalence classes of ideals. We have checked them in the exceptional groups.

Conjecture 6.1. Let $\mathcal{O}_{e}$ be a nilpotent orbit. Let $K$ be a subgroup of $A(e)$. There is at most one equivalence class $[I]$ such that $\mathcal{O}_{I}=\mathcal{O}_{e}$ and $K_{I}=K$.

Corollary 3.2 shows that if there are two equivalence classes $[I]$ and $[J]$ with $\mathcal{O}_{I}=\mathcal{O}_{J}$ and $[I] \preceq[J]$, then $K_{I} \subseteq K_{J}$. It seems likely that the converse holds. Namely,

Conjecture 6.2. Given two ideals $I$ and $J$ with $\mathcal{O}_{I}=\mathcal{O}_{J}$ and $K_{I} \subseteq$ $K_{J}$, then $[I] \preceq[J]$. 
A key unresolved question is which subgroups of $A(e)$ arise as $K_{I}$ for some equivalence class $[I]$. From the work in [5] it is clear that these subgroups are closely related to the subgroups which arise as stabilizers in $A(e)$ of irreducible components of the Springer fiber of $e$, that is, the fiber over $e$ of the moment map

$$
G \times{ }^{B} \mathfrak{n} \longrightarrow \mathfrak{g}
$$

However, it is possible for these groups to differ. For example if $e$ is of type $A_{4}+A_{1}$ in $E_{8}$, then $A(e)=S_{2}$. There are two classes of ideals with $\mathcal{O}_{I}=\mathcal{O}_{e}$. One has $K_{I}=1$ and the other has $K_{I}=S_{2}$. On the other hand, the stabilizer in $A(e)$ of any irreducible component of the Springer fiber is trivial. It would be nice to understand better what is going on here. At the least it seems reasonable to conjecture

Conjecture 6.3. Let $I$ be an ideal and let $e \in \mathcal{O}_{I}$. Let $K=K_{I}$. Then all irreducible representations of $A(e)$ arising in $\operatorname{Ind}_{K}^{A(e)}(1)$ contribute to the Springer correspondence.

Let $\bar{A}(e)$ be Lusztig's quotient of $A(e)$. Given a conjugacy class $C$ in $\bar{A}(e)$ of $A(e)$, there is a corresponding subgroup of $\bar{A}(e)$ constructed in [11] and [2]. Let $H_{C}$ be the pre-image of this subgroup in $A(e)$.

Conjecture 6.4. Assume that $H_{C}$ satisfies the Springer condition in Conjecture 6.3. Then $H_{C}=K_{I}$ for some ideal I satisfying $\mathcal{O}_{I}=\mathcal{O}_{e}$.

Let $\mathcal{N}_{o, \bar{c}}$ denote the set of pairs consisting of a nilpotent element and a conjugacy class in $\bar{A}(e)$ (up to conjugation by $G$ ). Achar [1] defined a partial order on the set $\mathcal{N}_{o, \bar{c}}$ using the work in [17]. We speculate that the partial order on equivalence classes of ideals is almost enough to completely explain Achar's partial order on $\mathcal{N}_{o, \bar{c}}$.

Let $(e, C),\left(e^{\prime}, C^{\prime}\right)$ be elements of $\mathcal{N}_{o, \bar{c}}$ such that the subgroups $H_{C}$, $H_{C^{\prime}}$ satisfy the hypothesis of Conjecture 6.4. Assuming also the uniqueness property of Conjecture 6.1 , let $[I],\left[I^{\prime}\right]$ be the corresponding equivalence classes. Then

ConjeCture 6.5. $[I] \preceq\left[I^{\prime}\right]$ if and only if $(e, C) \preceq\left(e^{\prime}, C^{\prime}\right)$, where the latter is the partial order on $\mathcal{N}_{o, \bar{c}}$ defined in [1].

We have verified all of these conjectures in the exceptional groups using our explicit computations. This led us to some corrections in the tables of [1] 
for $E_{8}$ that must have propagated from the misprints mentioned above in [3]. Namely, the following are related in Achar's partial order: $\left(E_{8}\left(b_{5}\right), E_{7}\left(a_{2}\right)+\right.$ $\left.A_{1}\right) \leq\left(E_{7}\left(a_{1}\right), 1\right),\left(A_{3}, 1\right) \leq\left(A_{3}+A_{1}, 1\right),\left(E_{8}\left(b_{6}\right), D_{8}\left(a_{3}\right)\right) \leq\left(E_{6}+A_{1}, 1\right)$, and $\left(D_{4}\left(a_{1}\right), 3 A_{2}\right) \leq\left(D_{4}\left(a_{1}\right)+A_{1}, 3 A_{2}+A_{1}\right)$. There is also one redundant line starting at $\left(A_{4}+A_{2}, 1\right)$.

\section{Appendix: The partial order on equivalence classes in the excep- tional groups}
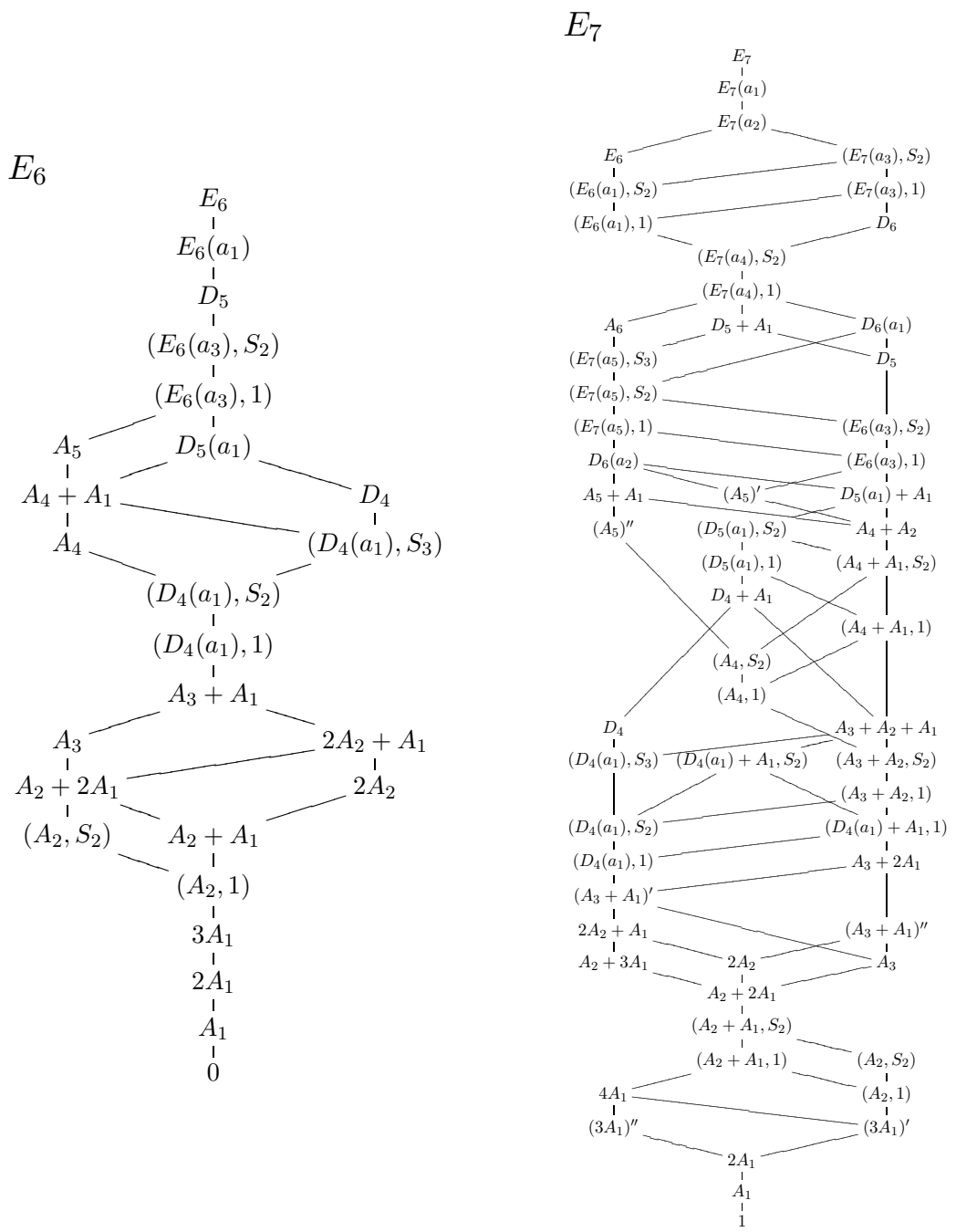
$E_{8}$

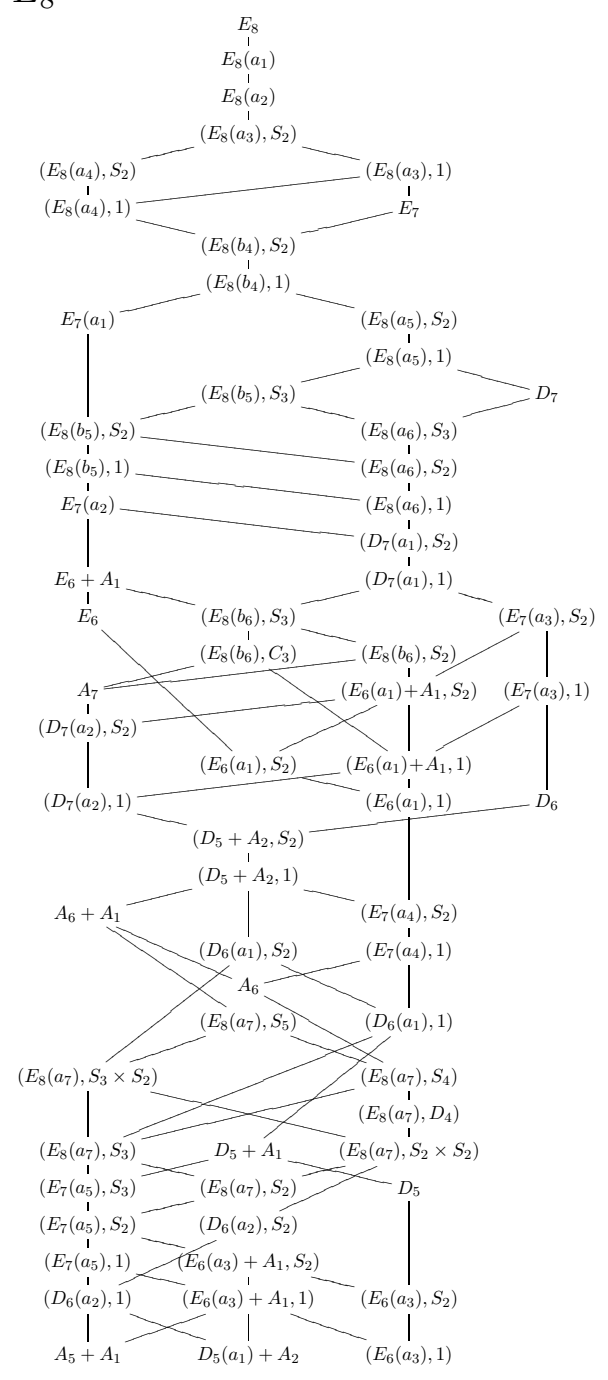

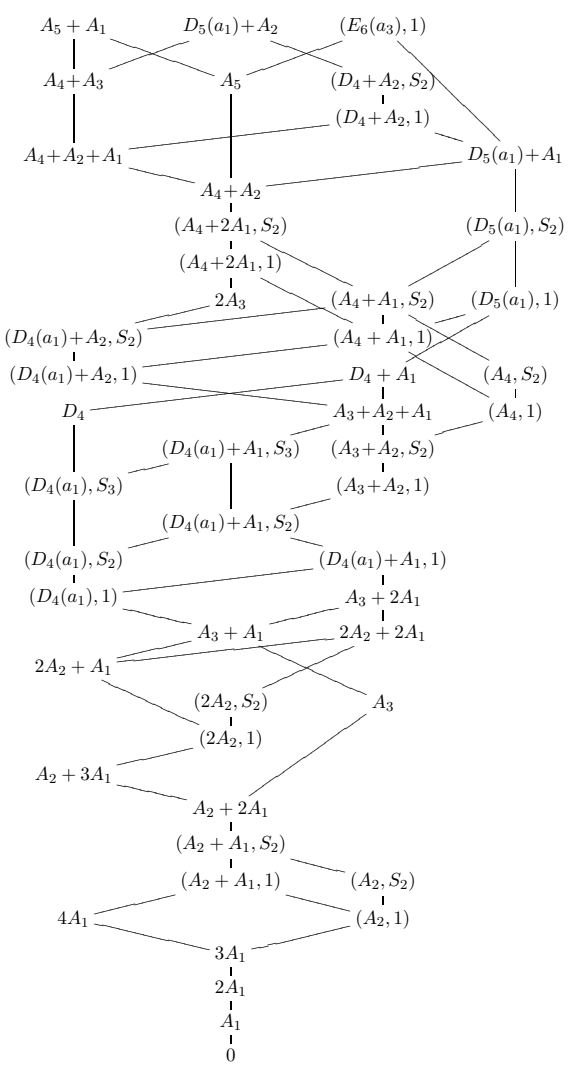




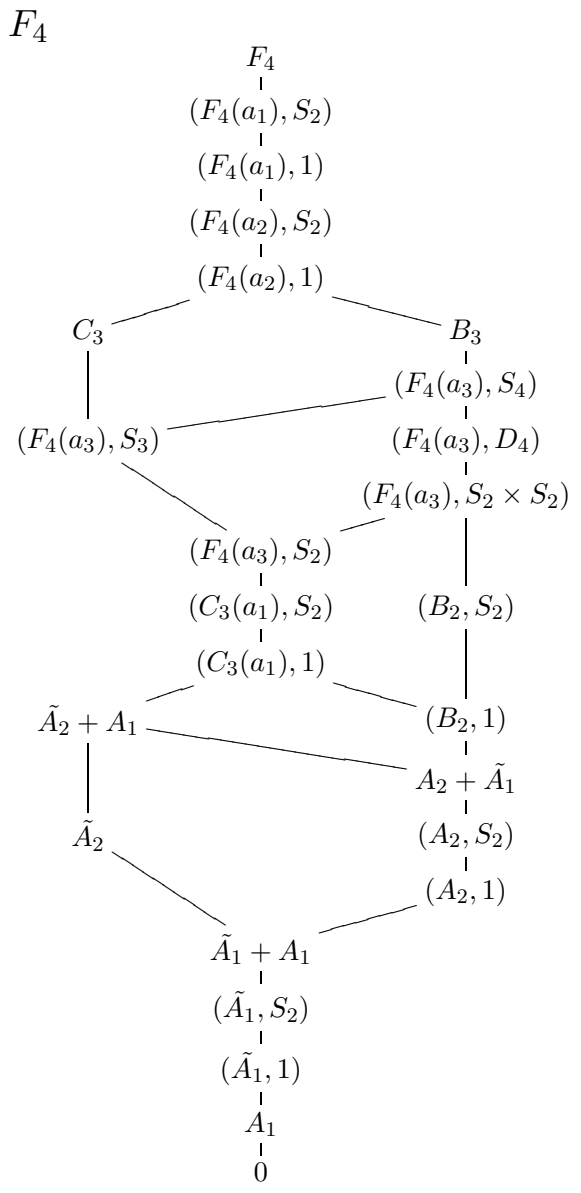

$G_{2}$


$\tilde{A}_{1}$ $A_{1}$

!

\section{REFERENCES}

[1] P. Achar, An order reversing duality for conjugacy classes in Lusztig's canonical quotient, Tranformation Groups, 8 (2003), no. 2, 107-145.

[2] P. Achar and E. Sommers, Local systems and functions on orbit covers, Representation Theory, 6 (2002), 190-201.

[3] R. Carter, Finite groups of Lie type. Conjugacy classes and complex characters, Wiley Classics Library, John Wiley \& Sons, Ltd, Chicester, 1993.

[4] D. H. Collingwood and W. M. McGovern, Nilpotent Orbits in Semisimple Lie Algebras, Van Nostrand Reinhold Mathematics Series, Van Nostrand Reinhold Co., New York, 1993.

[5] C. De Concini, G. Lusztig, and C. Procesi, Zero set of a nilpotent vector field, Journal of the A.M.S., 1 (1988), no. 1, 15-34.

[6] M. Demazure, A very simple proof of Bott's Theorem, Invent. Math., 33 (1976), 
$271-272$.

[7] J. C. Jantzen, Nilpotent orbits in representation theory, Lie Theory (Boston), Progr. Math., vol. 228, Birkhäuser, Boston (2004), pp. 1-211.

[8] N. Kawanaka, Generalized Gelfand-Graev representations of exceptional simple groups over a finite field I, 84 (1986), Invent. Math., 575-616.

[9] — Orbits and stabilizers of nilpotent elements of a graded semisimple Lie algebra, J. Fac. Sci. Univ. Tokyo, 34 (1987), 573-597.

[10] K. Liang and L. Lu, Sheets and rigid covers in the exceptional groups, Chinese Sci. Bull., 43 (1998), no. 20, 1702-1706.

[11] G. Lusztig, Notes on unipotent classes, Asian J. Math., 1 (1997), 194-207.

[12] G. Lusztig and N. Spaltenstein, Induced unipotent classes, J. London Math. Soc., 19 (1979), no. 1, 41-52.

[13] K. Mizuno, The conjugate classes of unipotent elements of the Chevalley groups $E_{7}$ and $E_{8}$, Tokyo J. Math., 3 (1980), no. 2, 391-461.

[14] D. Panyushev and G. Röhrle, On spherical ideals of Borel subalgebras, Arch. Math. (Basel), 84 (2005), no. 3, 225-232.

[15] J.-Y. Shi, The Kazhdan-Lusztig cells in certain affine Weyl groups, Lecture Notes in Mathematics, no. 1179, Springer-Verlag, 1986.

[16] E. Sommers, A generalization of the Bala-Carter theorem for nilpotent orbits, Internat. Math. Res. Notices (1998), no. 11, 539-562.

[17] Lusztig's canonical quotient and generalized duality, J. of Algebra (2001), no. $243,790-812$.

[18] Normality of nilpotent varieties in $E_{6}$, J. Algebra, 270 (2003), 288-306.

[19] B-stable ideals in the nilradical of a Borel subalgebra, Canadian Math. Bull., 48 (2005), no. 3, 460-472.

[20] N. Spaltenstein, Classes Unipotentes et Sous-Groupes de Borel, Lecture Notes in Mathematics, no. 946, Springer-Verlag, 1982.

[21] T. A. Springer, Linear Algebraic Groups, 2nd ed., Progress in Mathematics, Birkhäuser, Boston, 1998.

[22] R. Steinberg, Endomorphisms of linear algebraic groups, AMS, Providence, 1968.

University of Massachusetts - Amherst

Amherst, MA 01003

U.S.A.

esommers@math.umass . edu 